\title{
Recomendaciones conjuntas sobre la evaluación cardiovascular para la realización de ejercicio y deporte en personas menores de 35 años
}

\author{
Con el aval de la Sociedad Uruguaya de Cardiología (SUC) \\ y la Sociedad Uruguaya de Pediatría (SUP)
}

\author{
Redactores SUC: Dres. Sonia Rossi, Gerard Burdiat, Alejandro Cuesta, \\ Oscar Díaz Arnesto, Juan Carlos Gambetta \\ Redactores SUP: Dres. Catalina Pinchak, Suci Dutra, Pedro Chiesa, \\ Diego Abdala, Carla Papone, Giselle Pérez
}

\section{Resumen}

Se presentan, en un trabajo conjunto, las recomendaciones para la evaluación cardiovascular de personas menores de 35 años que realizan ejercicio y deportes.

Se establecen pautas en cuanto a la valoración y la habilitación que tendrá dicha evaluación, quiénes estarán a cargo de su realización y cuáles son los componentes fundamentales de una correcta valoración cardiovascular. Se toman en cuenta los antecedentes familiares y personales, el examen físico exhaustivo y se considera conveniente la realización del electrocardiograma como parte integrante obligatorio. Se hacen referencia a otros estudios funcionales como opcionales, así como la posible derivación a los especialistas cuando se considere necesario.

Palabras clave: EVALUACIÓN

EJERCICIO

DEPORTE

ELECTROCARDIOGRAMA

\section{Recommendations for cardiovascular evaluation of people under 35 years of age who perform exercise and sports are presented in a joint work}

\section{Summary}

Guidelines are established regarding the assessment and qualification that said evaluation will have, who will be in charge of carrying it out and what are the fundamental components of a correct cardiovascular evaluation. The family and personal history and an exhaustive physical examination are taken into account and the performance of the electrocardiogram is considered advisable as a mandatory integral part. Reference is made to other functional studies as optional as well as possible referral to specialists when deemed necessary.

Key words:

EVALUATION

EXERCISE

SPORT

ELECTROCARDIOGRAM

Correspondencia: suc@adinet.com.uy. Sitio web: www.suc.org.uy

Conflictos de interés: los autores declaran no tener conflictos de intereses.

Nota al editor: este artículo, como recomendación conjunta, se presenta para ser publicado en forma simultánea en la Revista Uruguaya de Cardiología y en la revista Archivos de Pediatría del Uruguay.

Recibido Oct 13, 2021; aceptado Nov 11, 2021 


\section{As recomendações para a avaliação cardiovascular de menores de 35 anos que praticam exercícios e esportes são apresentadas num trabalho conjunto}

\section{Resumo}

Estabelecem-se as diretrizes quanto à avaliação e qualificação que terá essa avaliação, quem se encarregará de realizá-la e quais são os componentes fundamentais de uma correta avaliação cardiovascular. A história familiar e pessoal, o exame físico exaustivo são levados em consideração e a realização do electrocardiograma é considerada aconselhável como parte integrante obrigatória. É feita referência a outros estudos funcionais como opcional, bem como possível encaminhamento para especialistas quando considerado necessário.

Palavras chave: AVALIAÇÃO

EXERCÍCIO

ESPORTE

ELECTROCARDIOGRAMA

\section{Principales aportes}

En un trabajo conjunto entre sociedades médico-científicas, se consensuan una serie de recomendaciones sobre la evaluación cardiovascular previa a la realización de ejercicio y deportes en personas menores de 35 años. Este documento, inédito en nuestro país hasta el momento, pretende aportar a la elaboración de futuras guías de evaluación predeportiva, y, a la vez, ser un insumo de primera mano para las autoridades ministeriales correspondientes y estatales

El movimiento regular, la actividad física y el deporte han demostrado ser beneficiosos para la salud y prolongar la vida. Evitar el comportamiento sedentario debe ser fuertemente recomendado a toda la población. Esa recomendación general debe luego aplicarse a cada caso individual, con la mejor evidencia disponible, teniendo en cuenta la edad, la situación física, psíquica y el grado de entrenamiento, entre otras.

La evaluación del estado de salud de las personas que realizan ejercicio, deporte recreativo y/o competitivo involucra múltiples aspectos de su situación biológica, psicológica y social. La salud cardiovascular y en particular la identificación de condiciones que pueden predisponer a la ocurrencia de muerte súbita son una parte de ello y es la que abordan estas recomendaciones.

La prevención de la muerte súbita relacionada con el deporte involucra varios aspectos: la valoración del estado de salud general y en especial cardiovascular, la seguridad en el ámbito deportivo, desde el clima hasta las instalaciones, la disponibilidad de personas entrenadas en resucitación cardiopulmonar básica y el acceso rápido a un desfibrilador externo automático. Todas son im- portantes, pero estas recomendaciones se centran en el primer aspecto.

Aquí se establecen recomendaciones para la valoración sistemática de las personas en aparente estado de salud que quieran realizar o estén realizando ejercicio de cualquier intensidad o deporte. No se refiere de manera exclusiva al atleta competitivo ni de alto rendimiento(1). Se excluye a los sujetos con patología cardiovascular u otra condición médica que implica la aplicación de medidas específicas a su situación, para lo cual existen pautas internacionales específicas y se pueden apoyar en especialistas ${ }^{(2-4)}$.

Estas recomendaciones no agotan el abordaje de los deportes de alto riesgo. Por lo tanto, no se considera aquí la indicación de interconsultas ni estudios en personas que realizan estos deportes de forma competitiva y/o buscando un alto rendimiento ${ }^{(5)}$.

Estas recomendaciones se realizan para dos grupos etarios: de 6 a 12 años y desde 12 años cumplidos hasta 35 años. Para las personas mayores de esos rangos, se deben tener en cuenta otras consideraciones especiales que no son tratadas en estas recomendaciones.

\section{La valoración y la habilitación para hacer ejercicio o deporte se debe realizar cada un período máximo de 2 años.}

Varias de las condiciones patológicas y predisponentes que se buscan tienen un carácter evolutivo, por lo que la valoración debe renovarse en forma periódica. Esto no condiciona la aplicación de frecuencias menores o mayores por otras áreas de la salud y/o por deportes de intensidades o tipos particulares $^{(5-7)}$.

La valoración y la habilitación para el ejercicio o deporte deben ser realizadas por el pediatra, el médico de familia y/o el médico generalista (lo que corresponda en cada caso y ámbito 
institucional en que tenga lugar). Debe llevarse a cabo en el marco de los controles periódicos integrales de salud que se realizan a toda la población.

Teniendo en cuenta la buena disponibilidad de profesionales médicos y los niveles de cobertura nacional del Sistema Nacional Integrado de Salud, no es necesario que la valoración para el deporte quede a cargo de otros profesionales de la salud, sin perjuicio de que el médico pueda trabajar en conjunto con otros especialistas.

La valoración para el ejercicio y el deporte podrá ser realizada también por un médico especialista en deporte, pero ello no sustituye al control integral de salud mencionado.

Dado el número de personas a evaluar, la necesaria integralidad de esa evaluación y procurando facilitar el acceso a la actividad física, no es deseable la instrumentación de programas generales que supongan la consulta obligada con otros especialistas, salvo los casos en que el médico de referencia lo entienda justificado. En esos casos, se debe tener fácil y rápido acceso a los especialistas que los médicos evaluadores pudieran requerir: deportólogo, cardiólogo, cardiólogo pediatra según la edad, etcétera.

\section{La valoración y la habilitación para el ejercicio \\ y el deporte deben incluir la valoración \\ cardiovascular.}

La valoración de los deportistas dirigida a evaluar su salud cardiovascular y la prevención de la muerte súbita ha demostrado bajar significativamente la ocurrencia de eventos adversos. Esto es así cuando la valoración consta de interrogatorio, examen físico y la realización de un electrocardiograma (ECG) basal. La inclusión del ECG en la valoración aumenta por 6 la capacidad de detectar anormalidades y, además, es más económico en términos de la cantidad de casos detectados en relación al dinero invertido ${ }^{(6-8)}$. Es por ello que ha sido incorporado por la mayoría de las organizaciones deportivas internacionales y países donde hay protocolos nacionales de evaluación ${ }^{(9-12)}$. Hay que decir que a nivel liceal y universitario en Estados Unidos no se incorporó aún el ECG. Las valoraciones son realizadas por personal de salud no médico y ello obedecería a la accesibilidad para toda la población en ese país.

En Uruguay la normativa vigente ya recomienda la realización del ECG en la primera consulta a partir de los 12 años, pero lo deja a criterio del médico de referencia su aplicación ${ }^{(5)}$. La independencia profesional es un bien a atender, pero la práctica queda expuesta a las pautas que instrumenten las autoridades del prestador. Esto puede resultar en inequidad en el acceso en niños y adultos evaluados y no se ajusta a la evidencia argumentada y a los recursos disponibles en nuestro país.

La valoración y la habilitación para el ejercicio y el deporte de las personas de 12 años o más debe incluir la realización de interrogatorio y examen físicos orientados y electrocardiograma basal de 12 derivaciones.

La necesidad de ECG no supone la consulta con un cardiólogo. En la actualidad, en muchos casos, será necesario que el ECG sea informado por un cardiólogo y con experiencia en el diagnóstico electrocardiográfico en el corte etario que se considera. Estas recomendaciones suponen asumir un compromiso con la formación en el análisis e interpretación del ECG preventivo del sujeto sano y del deportista de todos los médicos involucrados. Es deseable que en el futuro todos los médicos del primer nivel de atención y/o que realizan controles periódicos integrales de salud puedan realizar la valoración y la habilitación en un solo tiempo.

La valoración y la habilitación para el ejercicio y el deporte de niños entre 6 y 12 años debe incluir la realización de interrogatorio y examen físicos orientados. Aquellos niños que realicen actividad física competitiva federada o que insuma 6 o más horas semanales deberán ser valorados también con electrocardiograma de 12 derivaciones.

Expertos cardiólogos pediatra y deportólogos desde la SUP están de acuerdo en realizar esta recomendación, si bien se reconoce que hay escasa bibliografía al respecto ${ }^{(13,14)}$.

\section{Interrogatorio y examen}

El interrogatorio y el examen físico es conveniente que sean realizados de forma sistemática y asistida por material impreso, como formulario codificado o lista de verificación. Recomendamos que allí se incluyan los denominados 14 pasos de la Asociación Americana del Corazón que pasamos a enumerar ${ }^{(0,15)}$ :

\section{Antecedentes personales}

1. Dolor torácico anterior u opresión que aparece en el esfuerzo y cede con el reposo (características de ángor típico).

2. Síncope o presíncope de causa inexplicada, que aparece durante el esfuerzo físico y/o en el cual no se sospecha origen reflejo. El síntoma en el posesfuerzo inmediato de un trabajo intenso suele ser de origen reflejo. 


\begin{tabular}{|c|c|}
\hline Variante normal en el ECG basal & Definición de la variante \\
\hline \multicolumn{2}{|c|}{$\begin{array}{l}\text { La presencia de estos signos, que pueden llamar la atención en otro contexto, deben ser considerados variantes } \\
\text { normales en personas que realizan o han realizado ejercicio físico o deporte de forma regular. No son signos de } \\
\text { alarma, aunque se presenten más de uno de ellos. }\end{array}$} \\
\hline $\begin{array}{l}\text { Voltajes aumentados como criterio de hipertrofia } \\
\text { ventricular derecha y/o izquierda }\end{array}$ & $\begin{array}{l}\text { Cualquiera de los criterios de uso habitual para defi- } \\
\text { nir hipertrofia ventricular por voltajes del QRS, dere- } \\
\text { cho y/o izquierdo. }\end{array}$ \\
\hline Bloqueo incompleto de rama derecha & $\begin{array}{l}\text { Presencia de onda R' en V1 con duración del QRS < } \\
120 \text { ms, se acompaña de patrón QRS en V6. }\end{array}$ \\
\hline Repolarización precoz & $\begin{array}{l}\text { Elevación del punto J, elevación del segmento ST y } \\
\text { ondas J en derivaciones inferiores o laterales. }\end{array}$ \\
\hline Variante normal en afrodescendientes & $\begin{array}{l}\text { Elevación del punto J, elevación del segmento ST } \\
\text { supraconvexo seguido de inversión de onda T hasta } \\
\text { V4 inclusive. }\end{array}$ \\
\hline Variante normal en adolescentes $<16$ años & Inversión de onda T hasta V3 inclusive. \\
\hline Bradicardia sinusal $\leq 30 \mathrm{cpm}$ & $\begin{array}{l}\text { También tener como normal la variabilidad respirato- } \\
\text { ria sinusal. }\end{array}$ \\
\hline Ritmo auricular no sinusal (ectópico) & $\begin{array}{l}\text { Ondas P con forma y eje que no corresponde a origen } \\
\text { sinusal; lo más frecuente son las P negativas en cara } \\
\text { inferior-ritmo auricular bajo. Cuando la frecuencia es } \\
>100 \text { cpm es una taquicardia auricular. }\end{array}$ \\
\hline Ritmo de la unión auriculoventricular & $\begin{array}{l}\text { Ritmo de QRS angosto < } 100 \text { cpm que tiene relación } \\
\text { variable con la despolarización auricular. Cuando hay } \\
\text { ritmo sinusal, este es más lento y así se ve el ritmo } \\
\text { de la unión, disociado y/o compitiendo con el sinusal. } \\
\text { Cuando hace un ejercicio y aumenta la frecuencia del } \\
\text { ritmo sinusal, desaparece el ritmo de la unión. }\end{array}$ \\
\hline Bloqueo auriculoventricular de $1^{\text {er }}$ grado & Considerar normal un intervalo PR $<400 \mathrm{~ms}$. \\
\hline $\begin{array}{l}\text { Intervalo PR corto con duración del QRS normal sin } \\
\text { onda Delta }\end{array}$ & $\begin{array}{l}\text { Muy frecuente verlo en jóvenes, pero también adultos. } \\
\text { Puede verse con un ritmo auricular no sinusal, por } \\
\text { ejemplo, un ritmo auricular bajo. }\end{array}$ \\
\hline Bloqueo auriculoventricular $2^{\circ}$ grado tipo 1 & $\begin{array}{l}\text { Intervalo PR se alarga hasta que una onda P no se con- } \\
\text { duce. En cada serie, el PR del último latido conducido } \\
\text { siempre es mayor que el PR del primero que se conduce. }\end{array}$ \\
\hline
\end{tabular}

3. Disnea o fatiga excesiva e inexplicada en relación con el ejercicio. Tiene especial importancia cuando aparece en un sujeto que viene entrenado y menos importancia cuando es de inicio de la práctica de ejercicio.

4. Detección de soplo cardíaco que no haya sido estudiado y desestimado.

5. Hipertensión arterial diagnosticada y/o tomando medicación antihipertensiva.

6. Restricción previa a deportes indicada por otro equipo médico.

7. Estudios cardiológicos previos pendientes o de los que desconocemos los resultados indicados por otro equipo médico.

\section{Antecedentes familiares}

8. Muerte inexplicada y repentina (muerte súbita) antes de los 50 años en algún familiar.

9. Discapacidad por causa cardíaca en familiar de $1^{\text {er }}$ grado $<50$ años.
10. Antecedente personal de patología cardíaca conocida como:

- cardiopatía hipertrófica o dilatada,

- síndrome de QT largo u otras canalopatías,

- trastorno del ritmo severo,

- síndrome de Marfan, y

- alteraciones genéticas cardíacas en familiares.

\section{Examen físico}

11. Soplo cardíaco, con signos de organicidad. Los soplos sistólicos en foco aórtico y pulmonar, que varían con la inspiración y espiración, de jóvenes delgados son en general soplos "funcionales".

12. Valoración de pulsos femorales: ausencia.

13. Valoración de estigmas de síndrome de Marfan.

14. Toma de presión arterial en miembros superiores e inferiores: diferencia $>20 \mathrm{mmHg}$ entre ambos miembros superiores o inferiores menor que superiores. En cualquier caso, verificar. 


\begin{tabular}{|c|c|}
\hline Alteración en el ECG basal & Definición de la alteración \\
\hline \multicolumn{2}{|c|}{$\begin{array}{l}\text { Las alteraciones que aquí se describen son poco esperables en quienes realizan ejercicio o deporte, pero solo la } \\
\text { presencia de dos o más de estos signos debe ser considerada como criterio de alarma, a efectos de avanzar en el } \\
\text { estudio de quien se trate. }\end{array}$} \\
\hline Eje QRS desviado a extrema izquierda & Entre $-30^{\circ}$ a $-90^{\circ}$ \\
\hline Eje QRS desviado a extrema derecha & $>120^{\circ}$. \\
\hline Sobrecarga auricular izquierda & $\begin{array}{l}\text { Onda } \mathrm{P}>120 \mathrm{~ms} \text { de duración en DI o DII con compo- } \\
\text { nente negativo de la onda P bifásica en V1 }>1 \mathrm{~mm} \text { x } \\
40 \mathrm{~ms} \text {. }\end{array}$ \\
\hline Sobrecarga auricular derecha & Onda P > 2,5 mm en DII, DIII y/o aVF. \\
\hline Bloqueo de rama derecha (BCRD) & QRS $\geq 120$ ms con la morfología típica. \\
\hline
\end{tabular}

\section{Electrocardiograma}

\section{Para el análisis y la interpretación del} electrocardiograma se recomienda utilizar los criterios electrocardiográficos de Seattle refinados, también denominados criterios de Sharma ${ }^{(16)}$. El uso de esos criterios ha demostrado mejorar la especificidad sin bajar la sensibilidad. Evitará demoras en la habilitación de personas que son sanas y solo presentan características propias de la edad y/o modificaciones propias del corazón de deportista. Su utilización sistemática simplifica mucho el informe de los ECG y su enseñanza ${ }^{(16,17)}$.

El conocimiento general sobre el ECG basal es necesario para su interpretación. A efectos de estas recomendaciones, tienen especial jerarquía la identificación de signos de alarma que pueden ponernos en la pista de enfermedades que predispongan a la muerte súbita, debiendo además diferenciarlos de las modificaciones normales del corazón del deportista. Esto se hace con base en criterios epidemiológicos que luego se aplican a la persona en particular y su clínica.

Modificando las tablas de la publicación de Sharma y col. ${ }^{(16)}$, se presentan criterios electrocardiográficos a los que se debe prestar especial atención. Hay signos que deben ser considerados normales en quienes realizan actividad física importante (tabla 1), signos que solo deben ser considerados de alarma si se están presentes más de uno asociados (tabla 2) y signos que por sí solos son de alarma y deben llevar al diagnóstico de ECG anormal (tabla 3).

\section{Estudios de imagen}

Para la valoración y la habilitación para

el ejercicio y deporte NO está indicada la realización de estudios de imagen en ninguna persona que no presente criterios de riesgo en la clínica y el electrocardiograma.

El ecocardiograma como herramienta de tamiza- je de primera línea supone un costo adicional. No hay evidencia a nivel poblacional de que tenga un valor diagnóstico adicional en personas asintomáticas y sin factores de riesgo. Puede aumentar los falsos positivos y generar aún más estudios por hallazgos de dudoso valor diagnóstico ${ }^{(9)}$.

La indicación de estudios de imagen debe darse en el marco de un estudio dirigido a buscar patologías específicas y por quienes estén familiarizados con el manejo de estas.

\section{Ergometría}

Para la valoración y la habilitación para el ejercicio y el deporte NO está indicada la realización de ergometría en menores de 35 años que no presentan criterios de riesgo en la clínica y el electrocardiograma.

La indicación debe darse en el marco de un estudio dirigido a buscar patologías específicas y por quienes estén familiarizados con el manejo de estas ${ }^{(9)}$.

Aquí no está considerada la indicación de este tipo de estudio en deportistas de competencia, por otras razones que no sean la prevención de la muerte súbita. La medición de la capacidad de ejercicio, consumo de oxígeno máximo, etc., pueden ser de interés para especialistas, por ejemplo, deportólogos o neumólogos.

\section{Criterios para derivar a cardiólogo}

La presencia de alguno de los 14 puntos mencionados en interrogatorio y examen físico y/o el hallazgo de alguno de los elementos mencionados en los criterios electrocardiográficos supondrán avanzar más para descartar o arribar a un diagnóstico. En ese caso se recomienda la consulta con cardiólogo, con el ECG realizado y antes de solicitar otro tipo de estudios indicados por la razón que se lo deriva. 
Tabla 3. Anormalidades del ECG basal en deportistas entrenados, que siempre deben ser considerados como signos de alarma.

Anormalidad en el ECG basal

Definición de la anormalidad

Las anormalidades que aquí se describen NO son esperables y su presencia debe ser siempre considerada como signo de alarma a efecto de avanzar en el estudio de la persona evaluada.

Inversión onda T $\quad \geq 1 \mathrm{~mm}$ en dos o más derivaciones que NO sean aVR, DIII y V1.

Anterior

No afroamericanos Ya desde V2 en adelante es anormal.

Afroamericanos Si tienen inversión de la T precedido de elevación de punto J y ST supraconvexo, se acepta; es normal hasta en V4.

Menores de 16 años

Se acepta inversión de la T hasta V3 o T bifásica solo en V3 como normal.

Lateral DI y aVL y/o V5 y/o V6 (con una sola alcanza).

Inferior DII y aVF.

Inferolateral

DII y aVF y lateral.

Depresión del ST Infradesnivel $\geq 0,5 \mathrm{~mm}$ en 2 o más derivaciones contiguas.

Ondas Q $\mathrm{Q} / \mathrm{R}>0,25$ o $\mathrm{Q}>40 \mathrm{~ms}$ en 2 o más derivaciones (excluida DIII y aVR). Solo una amplitud de $2-3 \mathrm{~mm}$ de una onda $\mathrm{Q}$ de $<40 \mathrm{~ms}$ NO es criterio. Si Q solo está presente en V1 y V2, repetirlas asegurándose la correcta posición.

Bloqueo de rama izquierda (BCRI) QRS $\geq 120$ ms con la morfología típica, aunque sea intermitente.

Alteración inespecífica de la conducción intraventricular

Onda épsilon

Preexcitación ventricular

Intervalo QT prolongado

Hombres

Mujeres

Patrón de Brugada tipo 1

Bradicardia sinusal

Pausas sinusales

Bloqueo AV $1^{\circ}$ grado

Bloqueo $\mathrm{AV} 2^{\circ}$ grado tipo 2

Bloqueo AV completo

Arritmias supraventriculares

Extrasistolia ventricular

Arritmias ventriculares $\mathrm{QRS} \geq 140 \mathrm{~ms}$.

En V1 y V3.

$\mathrm{PR}<120 \mathrm{~ms}+$ onda Delta + QRS ancho. Las medidas del QRS normal varían con la edad. Solo PR corto NO es criterio de anormalidad.

QTc $\geq 470 \mathrm{~ms}$.

QTc $\geq 480 \mathrm{~ms}$.

Elevación inicio del ST $\geq 2 \mathrm{~mm}$, descenso supraconvexo, seguido de onda T negativa y simétrica en 1 o más de V1 a V3. $<30 \mathrm{cpm}$.

$\geq 3$ segundos. Solo con $\mathrm{PR} \geq 400 \mathrm{~ms}$ se debe considerar anormal. PR fijos y alguna(s) P no conducida(s).

Mucho cuidado en diferenciarlo de la disociación por frecuencia nodal mayor que sinusal, que no es anormal.

Taquicardia paroxística supraventricular, fibrilación, flutter.

Presencia de $\geq 2$ extrasístoles ventriculares en 10 segundos de trazado.

Presencia $\geq 1$ dupla o TV no sostenida.
Sonia Rossi, ORCID 0000-0001-5581-9644

Gerard Burdiat, ORCID 0000-0002-3338-9345

Alejandro Cuesta, ORCID 0000-0003-1315-5581

Oscar Díaz Arnesto, ORCID 0000-0001-9636-2206

Juan Carlos Gambetta, ORCID 0000-0002-5719-5289

Pedro Chiesa, ORCID 0000-0002-8943-8660

Catalina Pinchak, ORCID 0000-0001-7957-6341

Suci Dutra, ORCID 0000-0003-0759-2806

Diego Abdala, ORCID 0000-0002-5345-209X

Carla Papone, ORCID 0000-0003-4779-7005

Giselle Pérez, ORCID 0000-0003-3937-6518

\section{Bibliografía}

1. Maron BJ, Thompson PD, Ackerman MJ, Balady G, Berger S, Cohen D et al. Recommendations and considerations related to preparticipation screening for cardiovascular abnormalities in competitive athletes: 2007 update: a scientific statement from the American Heart Association Council on Nutrition, Physical Activity, and Metabolism: endorsed by the American College of Cardiology Foundation. Circulation. 2007; 115 (12): 164355. doi: 10.1161/CIRCULATIONAHA.107.181423. 
2. Pelliccia A, Sharma S, Gati S, Bäck M, Börjesson M, Caselli S et al. 2020 ESC Guidelines on sports cardiology and exercise in patients with cardiovascular disease. Eur Heart J. 2021; 42 (1): 1796. doi: 10.1093/eurheartj/ehaa605.

3. Maron BJ, Zipes DP. 36th Bethesda Conference: eligibility recommendations for competitive athletes with cardiovascular abnormalities. J Am Coll Cardiol. 2005; 45 (8): 131275. doi: 10.1016/j. jacc.2005.02.002.

4. Maron BJ, Chaitman BR, Ackerman MJ, Bayés de Luna A, Corrado D, Crosson JE et al. Recommendations for physical activity and recreational sports participation for young patients with genetic cardiovascular diseases. Circulation 2004;109 (22): 280716. doi: 10.1161/01.CIR.0000128363.85581.E1.

5. Anexos 1, 2 y 3 del Decreto n. ${ }^{\circ}$ 274/017 del Poder Ejecutivo del 25 de setiembre del 2017. [internet]. Montevideo: IMPO; 2017 [consultado el fecha]. Disponible en: https://www.impo.com.uy/ bases/decretos-originales/274-2017.

6. Corrado D, Basso C, Pavei A, Michieli P, Schiavon M, Thiene G. Trends in sudden cardiovascular death in young competitive athletes after implementation of a preparticipation screening program. JAMA. 2006; 296 (13): 1593601. doi: 10.1001/ jama.296.13.1593.

7. Corrado D, Pelliccia A, Bjørnstad HH, Vanhees L, Biffi A, Borjesson $M$ et al. Cardiovascular pre-participation screening of young competitive athletes for prevention of sudden death: proposal for a common European protocol. Consensus Statement of the Study Group of Sport Cardiology of the Working Group of Cardiac Rehabilitation and Exercise Physiology and the Working Group of Myocardial and Pericardial Diseases of the European Society of Cardiology. Eur Heart J. 2005; 26 (5): 51624 doi: 10.1093/eurheartj/ehi108.

8. Harmon KG, Suchsland MZ, Prutkin JM, Owens DS, Aukerman DF, Hwang CE et al. Comparison of cardiovascular screening in college athletes by history and physical examination with and without an electrocardiogram: efficacy and cost. Heart Rhythm. 2020; 17 (10): 164955. doi: 10.1016/j. hrthm.2020.04.032

9. Mont L, Pelliccia A, Sharma S, Biffi A, Borjesson M, Terradellas JB et al. Pre-participation cardiovascular evaluation for athletic participants to prevent sudden death: position paper from the EHRA and the EACPR, branches of the ESC. En- dorsed by APHRS, HRS, and SOLAECE. Europace. 2017; 19 (1): 13963. doi: 10.1093/europace/euw243.

10. Okuni M, Kusakawa S, Hozaki J, Hirayama T, Osano M, Murakami M et al. Development of a heart disease screening system for school children and its results in the Tokyo area in 1980. Jpn Circ J. 1982; 46 (11): 12504. doi: 10.1253/jcj.46.1250.

11. National Athletic Trainers' Association. The International Olympic Committee (IOC) consensus statement on periodic health evaluation of elite athletes: March 2009. J Athl Train. 2009; 44 (5): 53857. doi: 10.4085/1062-6050-44.5.538.

12. Steinvil A, Chundadze T, Zeltser D, Rogowski O, Halkin A et al. Mandatory electrocardiographic screening of athletes to reduce their risk for sudden death proven fact or wishful thinking? J Am Coll Cardiol. 2011; 57 (11): 12929. doi: 10.1016/j. jacc.2010.10.037.

13. Rodday AM, Triedman JK, Alexander ME, Cohen JT, Ip S, Newburger JW et al. Electrocardiogram screening for disorders that cause sudden cardiac death in asymptomatic children: a meta-analysis. Pediatrics. 2012; 129 (4): e9991010. doi: 10.1542/peds.2011-0643.

14. Vetter VL, Dugan N, Guo R, Mercer-Rosa L, Gleason M, Cohen $\mathbf{M}$ et al. A pilot study of the feasibility of heart screening for sudden cardiac arrest in healthy children. Am Heart J. 2011; 161 (5): 10001006.e3. doi: 10.1016/j.ahj.2011.01.022.

15. Maron BJ, Friedman RA, Kligfield P, Levine BD, Viskin S, Chaitman BR et al. Assessment of the 12-lead electrocardiogram as a screening test for detection of cardiovascular disease in healthy general populations of young people (12-25 years of age): a scientific statement from the American Heart Association and the American College of Cardiology. J Am Coll Cardiol. 2014; 64 (14): 1479514. doi: 10.1016/j.jacc.2014.05.006. Epub 2014 Sep 15. PMID: 25234655.

16. Sharma S, Drezner JA, Baggish A, Papadakis M, Wilson MG, Prutkin JM et al. International recommendations for electrocardiographic interpretation in athletes. Eur Heart J. 2018; 39 (16): 146680. doi: 10.1093/eurheartj/ehw631.

17. Sheikh N, Papadakis M, Ghani S, Zaidi A, Gati S, Adami PE et al. Comparison of electrocardiographic criteria for the detection of cardiac abnormalities in elite black and white athletes. Circulation. 2014; 129 (16): 163749. doi: 10.1161/ CIRCULATIONAHA.113.006179. 